\title{
Compósitos de policloreto de vinila e cortiça: avaliação das propriedades térmicas, inflamabilidade e morfologia
}

\author{
Pedro José Gomes Rodrigues ${ }^{1}$ \\ Lucas Rafael Carneiro da Silva ${ }^{1}$ \\ Arquimedes Lopes Nunes Filho ${ }^{1}$ \\ Renata Barbosa \\ Joyce Batista Azevedo ${ }^{2}$ \\ Tatianny Soares Alves $1 *$ (D)
}

\section{Resumo}

Os compósitos de cloreto de polivinil (PVC) e cargas vegetais têm sido amplamente utilizados em diferentes áreas em função da baixa densidade, melhores propriedades mecânicas e estabilidade dimensional. O presente trabalho teve como objetivo desenvolver compósitos de PVC/Cortiça com diferentes aspectos granulométricos e percentuais de 5 e $10 \%$. Os compósitos foram processados em uma extrusora monorosca, moldados por compressão em uma prensa hidráulica e avaliados quanto ao comportamento térmico por termogravimetria e teste de inflamabilidade vertical, e morfologia via microscopia óptica e eletrônica de varredura. Os resultados indicaram que, independentemente do conteúdo e tamanho dos grãos da cortiça, a estabilidade térmica dos compósitos não foi significativamente alterada. Quanto à inflamabilidade, todos os compósitos foram classificados como V-0, com tempo de extinção de chama menor que a matriz pura, com destaque para a composição com $10 \%$ de cortiça. Morfologicamente os compósitos apresentaram formação de aglomerados, poros e baixa adesão.

Palavras-chave: PVC; Cortiça; Compósitos; Comportamento térmico.

\section{Polyvinyl chloride and cork composites: evaluation of thermal, flammability and morphology}

\begin{abstract}
Composites of polyvinyl chloride (PVC) and vegetable fillers have been widely used in different areas due to their low density, better mechanical properties and dimensional stability. The present work had the objective of developing composites of PVC/Cork with load proportions, of different granulometric aspects, of 5 and $10 \%$. The composites were processed into a single screw extruder, then compression molded in a hydraulic press and evaluated for thermal behavior by thermogravimetry, vertical flammability test, and morphological characterization by optical microscopy and scanning electron. The results indicated that, regardless of the content and grain size of the cork, the thermal stability of the composites was not significantly altered. As for flammability, all composites were classified as V-0, with a flame extinction time lower than the pure matrix, especially in the presence of $10 \%$ of cork. Morphologically the composites showed formation of agglomerates, pores and low adhesion.
\end{abstract}

Keywords: PVC; Cork; Composites; Thermal behavior.

\section{Introdução}

O uso de recursos vegetais como cargas em polímeros termoplásticos tem sido o objetivo de pesquisas nas mais diversas áreas de pesquisa e aplicações industriais, como indústrias automobilísticas, embalagens, placas de montagem, painéis e móveis [1,2]. As possibilidades de associação entre melhores propriedades, redução de peso, emissão limitada

${ }^{I}$ Ciencia e Engenharia dos Materiais, Centro de Tecnologia, Universidade Federal do Piauí-UFPI, Teresina, PI, Brasil.

${ }^{2}$ Engenharia de Materiais, Universidade Federal do Recôncavo da Bahia-UFRB, Feira de Santana, BA, Brasil.

*Autor correspondente: tsaeng3@yahoo.com.br 
de dióxido de carbono e menor uso de fontes não renováveis de matérias-primas, são os principais elementos motivadores para o desenvolvimento de eco-compósitos [3]. Entre os materiais lignocelulósicos mais utilizados para esse fim estão a serragem, resíduos da indústria madeireira, fibras vegetais e resíduos agrícolas e agroindustriais lignocelulósicos [4-6]. Nos últimos anos compósitos a base de poli(cloreto de vinila) (PVC) e cargas vegetais ganharam destaque por apresentarem resistência térmica, estabilidade dimensional e baixa absorção de água [7].

O PVC é um polímero com características isolante (térmico, acústico e elétrico), de resistente à propagação de chamas, leve e que não se degrada por oxidação, sendo o segundo termoplástico mais consumido no mundo, com aplicações em diversos setores tais como construção civil e infraestrutura [8,9]. Obtido a partir de duas fontes primárias, sal marinho, responsável pelo fornecimento do cloro que corresponde a $57 \%$ em massa da cadeia polimérica, e o petróleo, que fornece o carbono e o hidrogênio, responsáveis por $43 \%$ do restante de massa [10]. Com característica versátil, o PVC quando aditivado produz compostos que variam desde um sólido rígido a um material de elevada flexibilidade, podendo ser incorporadas diferentes cargas, com destaque para as vegetais, possibilitando novas aplicações [9,11-13].

A cortiça é um material procedente da casca do sobreiro (Quercus suber L.), sendo composta por pequenas células que estão ligadas por uma resina tipicamente natural e com o interior de cada célula preenchido com ar. Em razão dessa estrutura celular, a cortiça apresenta-se como sendo um material compressível e que recupera a sua forma original após a remoção de uma força externa aplicada. Associando estas características, a cortiça é tida como um material ideal para isolamento elétrico, acústico e térmico, compressibilidade, absorção de vibrações, tendo potencial uso para a indústria de construção [14-18].

Neste contexto, o trabalho teve por objetivo o desenvolvimento de compósitos termoplásticos com PVC e cortiça, possibilitando a obtenção de um material de baixo custo, com uma carga biodegradável e de fonte renovável. Considerando que nenhum material reúne todas as características necessárias em todos os domínios de aplicação, os resultados deste trabalho podem contribuir para novas linhas de pesquisa em materiais compósitos, inserindo no contexto nacional uma carga pouco explorada.

\section{Materiais e métodos}

\subsection{Materiais}

Foi utilizado como matriz polimérica o PVC Poli(Cloreto de Vinila) - fabricado pela Karina Indústria e Comércio de Plásticos Ltda, grade BWF e lote 650922, aditivado com o antichama BWF-B. O polímero foi doado pela Indústria Copperline Fios e Cabos. Foi empregada como carga vegetal a cortiça granulada (\#1-2 mm) ou micronizada (\#74 $\mu \mathrm{m}$ ), fornecida pela Empresa Corticeira Paulista.

\subsection{Métodos}

\subsubsection{Processamento e caracterização dos compósitos}

A formulação dos compósitos PVC/Cortiça é apresentada na Tabela 1 .

Antes do processamento, a cortiça foi seca em estufa durante 24 horas à $60^{\circ} \mathrm{C}$. As proporções de cortiça foram definidas com base nas limitações das condições de processamento do equipamento utilizado, uma extrusora monorosca sem side feeder (alimentação lateral) e com base nos trabalhos de Fernandes et al. [19] e Vasconcelos et al. [20]. Os compósitos foram processados em extrusora monorosca modelo AX-16 da AX Plásticos, sob) e perfil de temperatura de $175-190{ }^{\circ} \mathrm{C}$, determinado a partir dos resultados da análise termogravimétrica da carga vegetal, e velocidade de rosca de $50 \mathrm{rpm}$. Seguido ao processo de extrusão foi realizada a secagem prévia dos compósitos à temperatura ambiente por um período de 2 dias. Removido o excesso de umidade, os compósitos foram peletizados e, em seguida, acondicionados em estufa sob temperatura de $70{ }^{\circ} \mathrm{C}$ durante $24 \mathrm{~h}$. Como parâmetro de comparação, o PVC puro foi extrusado nas mesmas condições dos compósitos.

A moldagem foi realizada por compressão em uma prensa hidráulica modelo MH-08-MN da MH Equipamentos, com dimensões preconizadas pela Norma ASTM D638 e UL $94 \mathrm{~V}$ para posterior caracterização. Os compósitos foram moldados sob compressão inicial de 0,5 ton. a $195^{\circ} \mathrm{C}$ durante 1 min e $30 \mathrm{~s}$, e compressão final de 2,5 ton. a $195^{\circ} \mathrm{C}$ por $4 \mathrm{~min}$.

A caraterização morfológica via MEV das superfícies de fratura dos compósitos, submetidos ao ensaio de resistência à tração, foi realizada com o intuito de avaliar a dispersão da cortiça na matriz polimérica, a adesão carga/matriz e formação de poros na estrutura. Os compósitos foram avaliados em microscópio Shimadzu modelo SSX-550 com voltagem de aceleração de feixe de elétrons de $15 \mathrm{kV}$, com diferentes faixas de ampliação de imagens.

A análise termogravimétrica, empregada para avaliar a temperatura de degradação de materiais, foi realizada sob atmosfera de oxigênio com fluxo de $100 \mathrm{~mL} \mathrm{~min}{ }^{-1}$, com

Tabela 1. Formulação dos compósitos

\begin{tabular}{lccc}
\hline Compósitos & PVC (\%) & $\begin{array}{c}\text { Cortiça em Pó } \\
(\%)\end{array}$ & $\begin{array}{c}\text { Cortiça } \\
\text { Granulada } \\
(\%)\end{array}$ \\
\hline PVC & 100 & 0 & 0 \\
PVC/5CP & 95 & 5 & 0 \\
PVC/5CG & 95 & 0 & 5 \\
PVC/10CP & 90 & 10 & 0 \\
PVC/10CG & 90 & 0 & 10 \\
\hline
\end{tabular}

Legenda: $\mathrm{CP}=$ Cortiça em Pó; $\mathrm{CG}=$ Cortiça Granulada. 
intuito de submeter os sistemas a condições mais severas de observação, taxa de aquecimento de $10{ }^{\circ} \mathrm{C} \mathrm{min}^{-1} \mathrm{e}$ com variação térmica de 32 a $1000^{\circ} \mathrm{C}$, em equipamento de marca SDT Q600 V 20.9 Build 20.

A avaliação do comportamento sob queima dos compósitos foi realizada de acordo com as condições estabelecidas pela Underwriters Laboratories (Norma UL 94) [21], teste de inflamabilidade vertical, em um total de 5 corpos de prova para cada composição.

A classificação final dos compósitos como V-0, V-1 ou V-2 foi realizada com base na determinação dos tempos (t1) e (t2) para que ocorra a extinção de chama após aplicação e reaplicação de uma fonte de queima, respectivamente, sendo estabelecido um tempo total de queima $(\mathrm{t} 1+\mathrm{t} 2)$, presença de gotejamento de material incandescente, combustão da camada de algodão, inserida abaixo da amostra, e propagação da chama até o suporte onde o corpo de prova é fixado.

Em síntese, a classificação é dita como V-0 quando a soma dos tempos decorridos para a extinção da chama no material não for superior a 50 s e a camada de algodão não entra em combustão devido ao gotejamento do material; V-1 quando a soma dos tempos $(\mathrm{t} 1+\mathrm{t} 2)$ não for superior a $250 \mathrm{~s}$ e não havendo queima do algodão por material desprendido. A classificação V-2 é semelhante a condição de tempo de extinção da chama, com diferencial para a condição de que a camada de algodão entra em queima pelo gotejamento do material.

A Microscopia Óptica foi utilizada com o intuito de caracterizar a morfologia dos compósitos de PVC/Cortiça antes e após a exposição à queima. As análises foram realizadas em microscópio óptico Leica Microsystems MD500 com câmera de captura ICC 50E, operando em modo reflexão. Foram capturadas imagens com ampliação de 40X (500 $\mu \mathrm{m})$ da superfície das amostras.

\section{Resultados e discussão}

A morfologia da cortiça em pó e granulada, e da superfície de fratura dos compósitos após o teste de tração é apresentada na Figura 1.
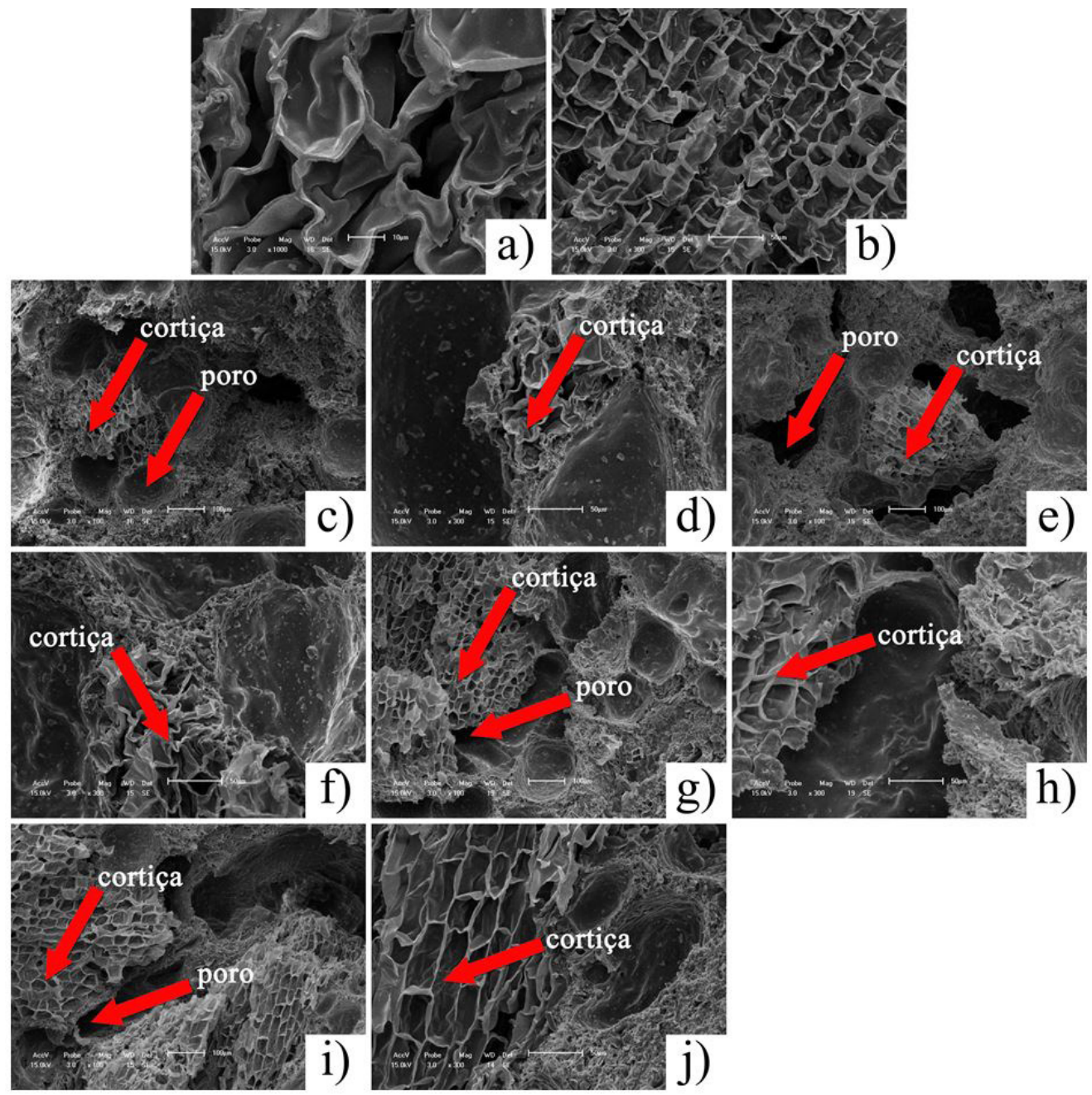

Figura 1. MEV da cortiça em pó (a); cortiça granulada (b); compósitos PVC/5CP (c) e (d); compósitos PVC/10CP (e) e (f); compósitos PVC/5CG (g) e (h); compósitos PVC/10CG (i) e (j). 
De acordo com as micrografias, foram observados nos compósitos de PVC/5CP e PVC/10CP aglomerados de carga na matriz, evidenciando a baixa eficiência de dispersão da cortiça na matriz de PVC. A pouca dispersão de carga foi provocada pela baixa eficiência em mistura do equipamento utilizado quando comparado, por exemplo, com uma extrusora dupla rosca corrotacional [22]. O mesmo comportamento foi observado para os compósitos $\mathrm{PVC} / 5 \mathrm{CG}$ e PVC/10CG.

A partir da análise morfológica de todos os sistemas, foi possível observar que os compósitos apresentaram pontos de baixa adesão entre as fases de polímero e cortiça. A interação entre os componentes, ainda que de baixa intensidade, pode ser atribuída a polaridade existente na estrutura química do PVC proporcionada pelo átomo de cloro, que interage com a estrutura da cortiça [23]. A cortiça é constituída principalmente de lignina (21-29\%), suberina $(33-45 \%)$ e polissacarídeos ou extrativos totais $( \pm 19 \%)$, em que, tanto a lignina quanto a suberina são compostos químicos de caráter majoritariamente polar, comportando-se de forma hidrofílica, favorecendo a interação entre o PVC e a cortiça $[24,25]$.

Outra característica comum aos compósitos foi a formação de poros, independente da granulometria e percentual de cortiça. A ausência de forças cisalhantes de alta intensidade, que pudessem favorecer um maior ancoramento mecânico da carga na matriz, a baixa adesão e, provavelmente, favoreceram a formação de porosidade nos compósitos.

Os resultados da análise termogravimétrica, apresentados na Figura 2, indicam que a decomposição térmica do PVC e dos compósitos ocorre em duas principais etapas, sob maiores temperaturas e com índices semelhantes. Para a matriz pura os perfis termogravimétricos apresentaram estágios iniciais de decomposição do PVC ocorrendo até $240{ }^{\circ} \mathrm{C}$, com pequena perda de massa (3\%) correspondendo a eliminação de umidade e a perda de ácido clorídrico $(\mathrm{HCl})$. Outro estágio de perda de massa foi observado na faixa entre $240-310{ }^{\circ} \mathrm{C}$ com a eliminação total do $\mathrm{HCl}$, provocando uma perda de massa de $37 \%$. Neste estágio acontece a formação de polienos, catalisando o processo de degradação da matriz [26]. O estágio entre $310-410{ }^{\circ} \mathrm{C}$ é representado pela resistência a quebra das ligações duplas dos polienos formados, promovendo pouca alteração na massa. Na segunda etapa de maior perda de massa, entre $410-480{ }^{\circ} \mathrm{C}$, foi observada uma perda de massa de $25 \%$, atribuída a cisão das sequências de polienos formados [27,28]. E por fim, entre $500-670{ }^{\circ} \mathrm{C}$ ocorre um estágio de decomposição que pode ser atribuído à presença dos aditivos plastificante e antichama BWF, com perda de $7 \%$ em massa. A degradação polimérica pode ocorrer por reação em cadeia e os subprodutos (hidroperóxidos) quebram a cadeia do polímero em segmentos de baixo peso molecular [29].

Os compósitos apresentaram perfil de degradação semelhante à matriz polimérica pura, independente do teor e granulometria da cortiça. Resultados semelhantes foram observados para compósitos de PVC e cargas vegetais [30,31]. De acordo com os resultados de análise térmica realizada em projetos do grupo de pesquisa com a mesma cortiça utilizada neste trabalho, Vasconcelos et al. [20] observaram que a degradação da cortiça natural, em níveis mais significativos, ocorreu a $278,44{ }^{\circ} \mathrm{C}$ e $409,10{ }^{\circ} \mathrm{C}$, com perda de massa $19,09 \%$ e $62,65 \%$, respectivamente, indicando elevada estabilidade térmica da carga vegetal empregada. Fernandes et al. [32] realizaram análise de degradação térmica dos principais constituintes da cortiça, lignina e suberina, e identificaram que a degradação de ambos ocorre de forma independente.

Em seus estudos, a suberina exibe boa estabilidade térmica até $260^{\circ} \mathrm{C}$, seguido de perda de massa progressiva, atingindo $45 \%$ em $338{ }^{\circ} \mathrm{C}$ e $87 \%$ em $494{ }^{\circ} \mathrm{C}$, e a lignina apresenta uma pequena perda a $193^{\circ} \mathrm{C}$ associada às estruturas monoméricas da lignina resultante da extração.

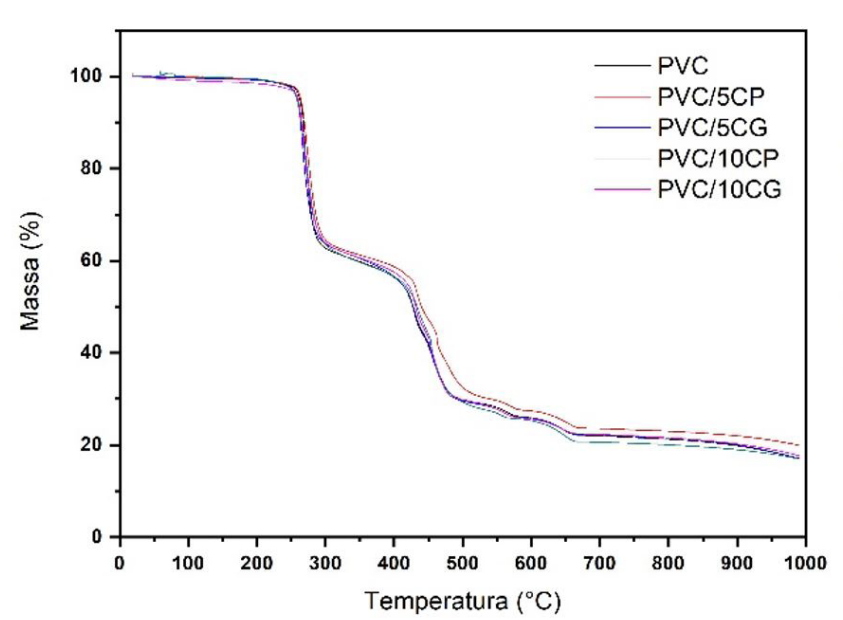

(a)

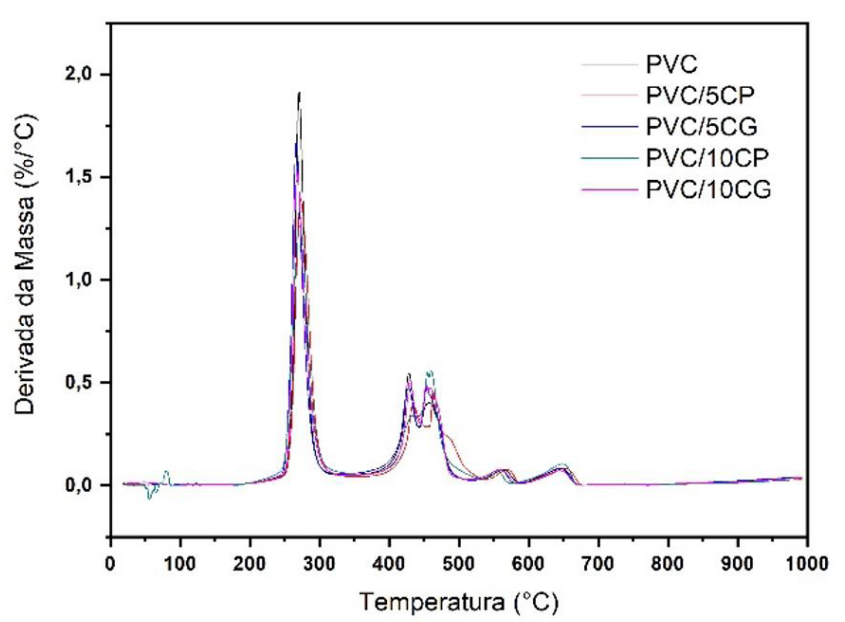

(b)

Figura 2. Curvas TG (a) e DTG (b) do PVC Puro e dos respectivos compósitos. 
Entre 300 e $400{ }^{\circ} \mathrm{C}$ ocorre a degradação dos polissacarídeos constituídos por celulose e hemicelulose, implicando em discretas variações de massa dos compósitos estudados [33]. Este comportamento evidencia que o fenômeno da degradação térmica é complexo e dominado pela presença do PVC e dependente da interação entre a matriz e a carga.

Becker et al. [34] avaliaram a degradação térmica dos compósitos de PVC rígido (PVCr) e fibras de bananeira (FB), e por análise termogravimétrica observaram que a adição das FB tratadas e não tratadas não alterou de forma significativa o comportamento térmico dos compósitos quando comparados a matriz de PVC Puro. Os autores atribuíram esse comportamento a fraca interação entre as fibras e a matriz, mesmo quando a carga foi submetida a tratamento químico, corroborando com os resultados obtidos.

Inicialmente, o comportamento sob chama foi avaliado segundo a Norma UL 94 HB [21] para a determinação dos seguintes parâmetros: velocidade média de propagação de chama, presença de gotejamento e a queima total do material durante o ensaio. Entretanto, devido a característica de supressão da chama após a retirada da fonte de calor não foi possível a determinação dos respectivos parâmetros. Esse tipo de comportamento é característico de polímeros como o PVC e nesta condição a avaliação foi realizada segundo a Norma UL $94 \mathrm{~V}$, com queima vertical [35]. Na Figura 3 são apresentados os resultados obtidos no teste de queima.

De acordo com os resultados obtidos, tempos de manutenção da chama e demais parâmetros de observação, as amostras de PVC Puro não apresentaram gotejamento de material e tiveram a chama suprimida após a remoção da fonte de calor, obtendo a classificação V-0. Este comportamento é justificado pela presença de átomo de cloro na estrutura conferindo ao material a característica inerente de auto-extinção. Por auto-extinção entende-se

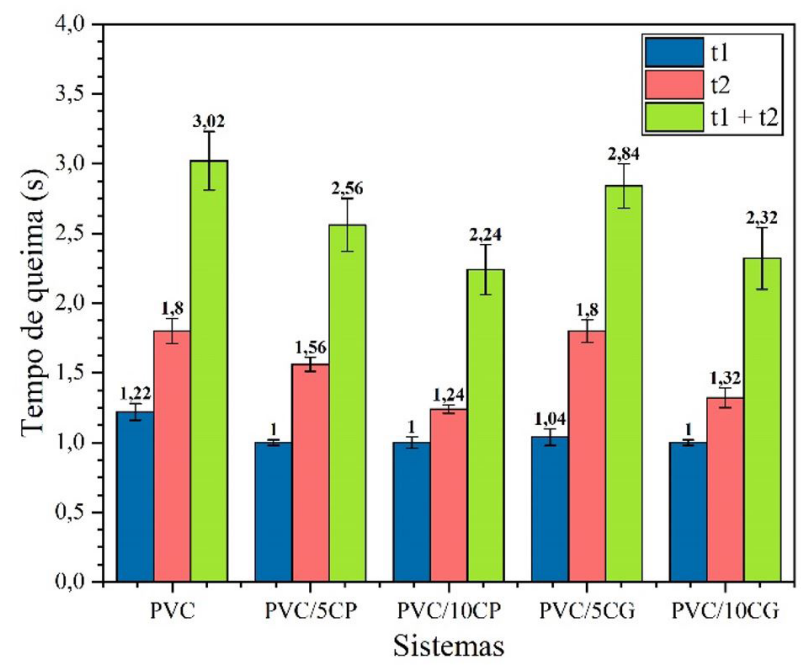

Figura 3. Resultado do teste de inflamabilidade vertical dos compósitos. a capacidade de um material de cessar a combustão imediatamente após a remoção da fonte de calor ou da chama [36].

Comparando o comportamento da resina pura e dos compósitos, a inserção da carga, de uma maneira geral, reduziu o tempo de manutenção da chama. Para os compósitos com cortiça em pó as reduções obtidas atingiram patamares de 15 e $25 \%$ para os sistemas $\mathrm{PVC} / 5 \mathrm{CP}$ e $\mathrm{PVC} / 10 \mathrm{CP}$, respectivamente. Com a inserção da carga granulada os compósitos PVC/5CG e PVC/10CG tiveram reduções de 6 e $23 \%$, indicando que a granulometria não foi um fator significativo para o processo de combustão do material. Essas reduções indicam que a carga auxiliou no processo de supressão da chama ao longo da amostra, possivelmente atuando como isolante térmico. Esse resultado pode ser atribuído a presença de suberina na composição da cortiça, que possui capacidade de isolamento, evitando a propagação mais extensa da chama e a elevada diferença do calor específico da carga e do polímero. Algumas estruturas lignocelulósicas apresentam comportamento de barreira contra o fogo, como é o caso do sobreiro que é mais resistente ao fogo do que outras árvores devido à lenta combustão da cortiça. A composição molecular e a estrutura fornecem a esse material a capacidade inerente de produzir resíduos carbonáceos termicamente estáveis quando expostos ao fogo [37]. No momento em que a chama incide sobre a amostra, a parte exterior fica recoberta por uma camada carbonizada atuando como barreira impedindo a difusão de oxigênio para o interior da amostra e com isso também dificultando o processo de combustão [38]. Esse comportamento foi destacado na Figura 4.

A formação de camadas carbonizadas é fundamental para o desenvolvimento de sistemas eficazes de retardantes de chama. Durante a combustão, os sistemas retardadores de chama carbonizados atuam criando uma camada isolante na superfície do material de combustão, resultando na melhoria de seu comportamento de fogo, reduzindo a difusão térmica e de oxigênio, bem como, a volatilização de produtos combustíveis [37]. Fernandes et al. [39] avaliaram a resistência ao fogo de compósitos de $\mathrm{PP} /$ Cortiça e PE/Cortiça e verificaram que os compósitos apresentaram um melhor comportamento de resistência a chama, permanecendo íntegros durante o teste. O pó de cortiça atuou como um retardador de fogo melhorando o desempenho dos compósitos. Outra vantagem quanto à utilização da cortiça na formação de compósitos poliméricos é a sua baixa emissão de monóxido e dióxido de carbono, de 0,1 a $0,6 \%$, e 0,1 a $2,3 \%$, respectivamente, quando comparada a incidência de gases tóxicos liberados pela matriz pura $[2,40]$ no momento da queima.

Diante desses resultados é possível observar que todos os compósitos apresentaram tempo de manutenção de chama inferior ao das amostras a base de PVC Puro, confirmando que a cortiça apresenta potencial de aplicação em sistemas com demanda de retardamento à chama. 


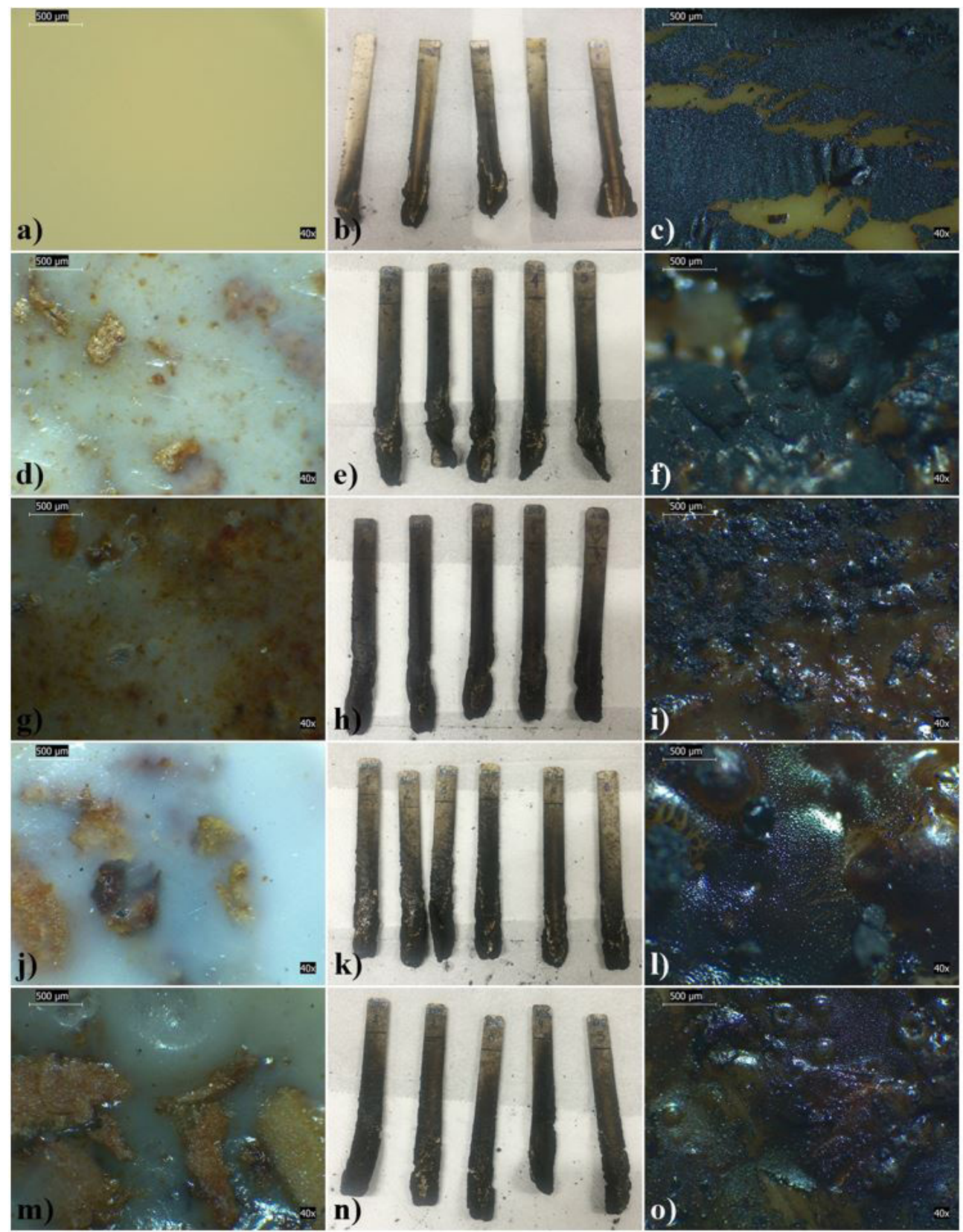

Figura 4. Microscopia óptica das superfícies das amostras antes do teste de queima PVC (a), PVC/5CP (d), PVC/10CP (g), PVC/5CG (j) e PVC/10CG (m); e após a finalização do ensaio PVC (c), PVC/5CP (f), PVC/10CP (i), PVC/5CG (1), PVC/10CG (o), e registro fotográfico dos corpos de prova ao final do ensaio de queima PVC (b), PVC/5CP (e), PVC/10CP (h), PVC/5CG (k), PVC/10CG (n).

\section{Conclusão}

Neste estudo foram desenvolvidos via extrusão compósitos de PVC/Cortiça em Pó e PVC/Cortiça Granulada e de acordo com os resultados obtidos os compósitos não apresentaram diferenças significativas entre os estágios de degradação térmica entre PVC puro e os compósitos.

Segundo os requisitos específicos da Norma UL 94 $\mathrm{V}$, o polímero puro e os respectivos compósitos obtiveram a classificação V-0, tendo os compósitos apresentado tempos de extinção de chama inferiores à matriz. Em geral, as propriedades analisadas apresentaram influência da presença da cortiça e morfologicamente apontaram ainda a necessidade de aplicação de agente de acoplamento e variações de condições de processamento de forma que as potencialidades dos compósitos preparados possam ser ampliadas.

\section{Agradecimentos}

Os autores agradecem ao CNPq e a FAPEPI pelo auxílio financeiro, a Copperline Fios e Cabos pela doação da resina $\mathrm{PVC}$. 


\section{Referências}

1 Catto AL, Dahlem MA Jr, Hansen B, Francisquetti EL, Borsoi C. Characterization of polypropylene composites using yerba mate fibers as reinforcing filler. Composites. Part B, Engineering. 2019;174:106935.

2 Silva JS, Catry F. Forest fires in cork oak (Quercus suber L.) stands in Portugal. The International Journal of Environmental Studies. 2006;63(3):235-257.

3 Andrzejewski J, Szostak M, Barczewski M, Łuczak P. Cork-wood hybrid filler system for polypropylene and poly(lactic acid) based injection molded composites: structure evaluation and mechanical performance. Composites. Part B, Engineering. 2019;163:655-668.

4 Eires R, Jalali S, Camões A. Novos compósitos eco-eficientes para aplicações não estruturais na construção. Revista Internacional Construlink. 2010;8:45-55.

5 Gil L. A cortiça como material de construção: manual técnico. Santa Maria de Lamas: Associação Portuguesa de Cortiça; 2007 [acesso em 6 jun. 2020]. Disponível em: https://www.apcor.pt/portfolio-posts/a-cortica-comomaterial-de-construcao-manual-tecnico

6 Balla VK, Kate KH, Satyavolu J, Singh P, Tadimeti JGD. Additive manufacturing of natural fiber reinforced polymer composites: processing and prospects. Composites. Part B, Engineering. 2019;174:106956.

7 Pulngern T, Chitsamran T, Chucheepsakul S, Rosarpitak V, Patcharaphun S, Sombatsompop N. Effect of temperature on mechanical properties and creep responses for wood/PVC composites. Construction \& Building Materials. 2016;111:191-198.

8 Souza MA, Pessan LA, Rodolfo A Jr. Nanocompósitos de poli (cloreto de vinila)(PVC)/argilas organofílicas. Polímeros. 2006;16(4):257-262.

9 Rodolfo A Jr, Mei LHI. Mecanismos de degradação e estabilização térmica do PVC: a review. Polímeros. 2007;17(3):263-275.

10 Carvalhais JCM. Estudo do comportamento de absorção de resinas de PVC com diferentes plastificantes [dissertação]. Coimbra: Universidade de Coimbra; 2013.

11 Dan-Asabe B. Thermo-mechanical characterization of banana particulate reinforced PVC composite as piping material. Journal of King Saud University-Engineering Sciences. 2018;30(4):296-304.

12 Doolittle AK. The technology of solvents and plasticizers. 1st ed. New York: Wiley; 1954.

13 Yang CG, Zeng HM, Li JJ. Fibre reinforced plastics. Compos. 1995;6:22.

14 Abdallah FB, Cheikh RB, Baklouti M, Denchev Z, Cunha AM. Characterization of composite materials based on PP-cork blends. Journal of Reinforced Plastics and Composites. 2006;25(14):1499-1506.

15 Sargianis J, Kim H-i, Suhr J. Natural cork agglomerate employed as an environmentally friendly solution for quiet sandwich composites. Scientific Reports. 2012;2(1):403.

16 Santos RG, Carvalho R, Silva ER, Bordado JC, Cardoso AC, Rosário Costa M, et al. Natural polymeric water-based adhesive from cork liquefaction. Industrial Crops and Products. 2016;84:314-319.

17 Walsh J, Kim H-I, Suhr J. Low velocity impact resistance and energy absorption of environmentally friendly expanded cork core-carbon fiber sandwich composites. Composites. Part A, Applied Science and Manufacturing. 2017;101:290-296.

18 Burley J, Evans J, Youngquist JA. Encyclopedia of forest sciences. 1st ed. Oxford: Academic Press; 2004.

19 Fernandes EM, Correlo VM, Mano JF, Reis RL. Polypropylene-based cork-polymer composites: processing parameters and properties. Composites. Part B, Engineering. 2014;66:210-223.

20 Vasconcelos GCMS, Carvalho LH, Barbosa R, Alves TS. Evaluation of the morphology, mechanical and thermal properties of cork and green polyethylene ecocomposites. Materials Research Express. 2019;6(9):095331.

21 Underwriters Laboratories. UL-94: test for flammability of plastic materials for parts in devices and appliances. Illinois: Underwriters Laboratories Inc.; 2001.

22 Manrich S. Processamento de termoplásticos: rosca única, extrusão e matrizes, injeção e moldes. 1. ed. São Paulo: Artliber; 2005.

23 Madaleno E, Rosa DS, Zawadzki SF, Pedrozo TH, Ramos LP. Estudo do uso de plastificantes de fontes renovável em composições de PVC. Polímeros. 2009;19(4):263-270. 
24 Moreira AC, Rios PDA, Vieira HC, Mori FA. Análise química da cortiça das árvores de Kielmeyera coriacea Mart. Revista Ciência da Madeira. 2017;8:1-9.

25 Fernandes EM, Correlo VM, Mano JF, Reis RL. Novel cork-polymer composites reinforced with short natural coconut fibres: effect of fibre loading and coupling agent addition. Composites Science and Technology. 2013;78:56-62.

26 Jordan KJ, Suib SL, Koberstein JT. Determination of the degradation mechanism from the kinetic parameters of dehydrochlorinated poly (vinyl chloride) decomposition. The Journal of Physical Chemistry B. 2001;105(16):31743181.

27 Pita VJRR, Monteiro EEC. Estudos térmicos de misturas PVC/plastificantes: caracterização por DSC e TG. Polímeros. 1996;6:50-56.

28 Del Carpio DCF. Degradação físico-quimica do PVC causada por derivados de petróleo [dissertação]. Rio de Janeiro: Pontifícia Universidade Católica do Rio de Janeiro; 2009.

29 Jakubowicz I. Evaluation of degradability of biodegradable polyethylene (PE). Polymer Degradation \& Stability. 2003;80(1):39-43.

30 Bai X-Y, Wang Q-W, Sui S-J, Zhang C-S. The effects of wood-flour on combustion and thermal degradation behaviors of PVC in wood-flour/poly (vinyl chloride) composites. Journal of Analytical and Applied Pyrolysis. 2011;91(1):34-39.

31 Fang Y, Wang Q, Guo C, Song Y, Cooper PA. Effect of zinc borate and wood flour on thermal degradation and fire retardancy of polyvinyl chloride (PVC) composites. Journal of Analytical and Applied Pyrolysis. 2013;100:230-236.

32 Fernandes EM, Aroso IM, Mano JF, Covas JA, Reis RL. Functionalized cork-polymer composites (CPC) by reactive extrusion using suberin and lignin from cork as coupling agents. Composites. Part B, Engineering. 2014;67:371-380.

33 Averous L, Boquillon N. Biocomposites based on plasticized starch: thermal and mechanical behaviours. Carbohydrate Polymers. 2004;56(2):111-122.

34 Becker D, Kleinschmidt AC, Balzer PS. Banana fibers and rigid PVC composites: effect of fiber treatment. Matéria. 2014;19(3):257-265.

35 Weil ED, Levchik S, Moy P. Flame and smoke retardants in vinyl chloride polymers-commercial usage and current developments. Journal of Fire Sciences. 2006;24(3):211-236.

36 Rodolfo A Jr, Nunes LR, Ormanji W. Tecnologia do PVC. 1. ed. São Paulo: Proeditores/Braskem; 2006.

37 Costes L, Laoutid F, Brohez S, Dubois P. Bio-based flame retardants: When nature meets fire protection. Materials Science and Engineering R Reports. 2017;117:1-25.

$38 \mathrm{Lu}$ R. Cork in the sector of construction. Lisboa: Instituto Superior Técnico; 2014 [acesso em 6 jun. 2020]. Disponível em: https://fenix.tecnico.ulisboa.pt/downloadFile/844820067123530/extended\%20abstract\%20final.pdf

39 Fernandes EM, Correlo VM, Chagas JAM, Mano JF, Reis RL. Properties of new cork-polymer composites: advantages and drawbacks as compared with commercially available fibreboard materials. Composite Structures. 2011;93:3120-3129.

40 Pausas JG. Resprouting of Quercus suber in NE Spain after fire. Journal of Vegetation Science. 1997;8(5):703-706.

Recebido em: 9 Abr. 2020

Aceito em: 5 Nov. 2020 